\title{
Rabaska
}

Revue d'ethnologie de l'Amérique française

\section{Chaire de recherche du Canada en patrimoine ethnologique (Université Laval)}

\section{Laurier Turgeon}

Volume 16, 2018

URI : https://id.erudit.org/iderudit/1051368ar

DOI : https://doi.org/10.7202/1051368ar

Aller au sommaire du numéro

Éditeur(s)

Société québécoise d'ethnologie

ISSN

1703-7433 (imprimé)

1916-7350 (numérique)

Découvrir la revue

Citer ce document

Turgeon, L. (2018). Chaire de recherche du Canada en patrimoine ethnologique

(Université Laval). Rabaska, 16, 333-339. https://doi.org/10.7202/1051368ar d'utilisation que vous pouvez consulter en ligne.

https://apropos.erudit.org/fr/usagers/politique-dutilisation/ 


\section{Chaire de recherche du Canada en patrimoine ethnologique}

6115, Pavillon Jean-Charles-Bonenfant

Université Laval

2345, allée des Bibliothèques

Québec (Québec)

G1V 0A6

Téléphone : (418) 656-7200

Courriel : Laurier.Turgeon@hst.ulaval.ca

Toile : www. chaire-patrimoine.ulaval.ca.

L'année 2017-2018 a été pour la Chaire de recherche du Canada en patrimoine ethnologique (CRCP) et son Laboratoire d'enquête ethnologique et de multimédia (LEEM) une année riche en projets et en expériences variées, tant en recherche fondamentale qu'en recherche appliquée. Le volet de recherche fondamentale de la Chaire a permis de mener une réflexion de fond sur l'histoire de l'ethnologie au Québec et au Canada français, et de préparer un article en anglais pour un ouvrage collectif consacré à l'histoire de l'ethnologie en Amérique du Nord dirigé par deux ethnologues américaines, Patricia Sawin et Rosemary Lévy Zumwalt, dans la prestigieuse collection d'ethnologie de la Indiana University Press. L'ouvrage regroupe une vingtaine de textes rédigés par des spécialistes nord-américains destinés à faire le bilan et à proposer des directions d'enseignements et de recherches en ethnologie. Le livre doit paraître à l'automne 2018. C'est surtout le volet de recherche appliquée et de recherche création qui a retenu l'attention du titulaire de la CRCP et de l'équipe du LEEM, notamment le projet du Corridor patrimonial, culturel et touristique du Canada.

\section{Le Corridor patrimonial}

La CRCP et son LEEM ont réalisé, de septembre 2016 à février 2018, le Corridor patrimonial, culturel et touristique francophone du Canada. Il s'agit d'un site web adaptatif, dont la taille s'ajuste à la dimension de l'écran de réception (ordinateur, tablette numérique ou téléphone intelligent) et d'une application mobile, téléchargeable par province et territoire, qui interprètent 150 sites patrimoniaux francophones du Canada ; ils se répartissent grosso modo en trois grandes régions : 50 dans les provinces de l'Atlantique, 50 au Québec et 50 en Ontario et dans l'Ouest.

Le projet a été guidé par un double objectif : d'abord, par la qualité scientifique de la documentation historique et ethnologique et, ensuite, par la mise en valeur du caractère vécu et vivant du patrimoine des francophones. Pour ce faire, la Chaire et son laboratoire ont mobilisé l'équipe de professionnels qui travaillent ensemble depuis une douzaine d'années, soit un historien (Martin Fournier), un ethnohistorien (Van Troi Tran) et deux ethnologues (Philippe Dubois, le coordonnateur du laboratoire, et Élise Bégin, une professionnelle travaillant à temps partiel), et une équipe de vidéastes, anciens étudiants de l'Université Laval, qui collaborent avec la Chaire depuis sa fondation (Helgi Paccinin, Michael Gravel et Benjamin Gadoury). À ce noyau dur, s'est ajoutée une demi-douzaine d'étudiants de $2^{\mathrm{e}}$ et de $3^{\mathrm{e}}$ cycles des programmes d'ethnologie et patrimoine de l'Université Laval, et trois stagiaires internationaux, dont deux du programme de master en patrimoine de l'Université Jean-Monnet de Saint-Étienne (France) et un du programme de master en mémoire et patrimoine de l'Université d'État d'Haïti. Dans un premier temps, l'équipe a voulu identifier les 150 sites ou éléments patrimoniaux francophones jugés les plus significatifs par les communautés francophones elles-mêmes. Des consultations ont été réalisées par 
téléphone et par courriel auprès des bureaux provinciaux du Réseau de développement économique et d'employabilité du Canada pour les communautés francophones et acadienne (RDÉE Canada) et d'une cinquantaine d'institutions, d'associations et de regroupements francophones partout au Canada. Dans un deuxième temps, nous avons constitué des dossiers documentaires et historiques sur les communautés francophones de chacune des provinces et sur les 150 sites ou éléments patrimoniaux sélectionnés. Dans un troisième temps, les équipes sont allées sur le terrain et ont mené des enquêtes ethnographiques auprès des représentants des organismes et des pratiquants de ces patrimoines dans toutes les provinces et tous les territoires du Canada. Nous avons réalisé plus de 80 entrevues filmées de 30 à 90 minutes partout où se trouvaient des francophones, par exemple à Yellowknife (Territoires du Nord-Ouest), à Whitehorse (Yukon), à Maillardville (Colombie-Britannique), au Lac La Biche (Alberta), à Régina (Saskatchewan), à Saint-Boniface (Manitoba), à Thunder-Bay (Ontario), à Québec (Québec), à Moncton (Nouveau-Brunswick), à Louisbourg (Nouvelle-Écosse), à Abram-Village (Île-du-Prince-Édouard) et à L'Anse-aux-Canards (Terre-Neuve). Ces entrevues filmées ont servi à alimenter le corpus documentaire du projet d'informations récentes et du terrain, et aussi à monter des vidéos courtes de 4 à 5 minutes illustrant de manière vivante les éléments des patrimoines francophones, notamment le patrimoine dit immatériel (fêtes, festivals, chanson, musique, danse, récits oraux, art, et artisanat). Tous ces éléments ont été réunis dans un programme interprétatif multimédia en ligne concis, cohérent et convivial.

Les patrimoines naturel, matériel et immatériel de ces sites sont interprétés à l'aide de cinq médias : le texte, la photo, l'enregistrement sonore, la vidéo et l'animation 3D. Le texte écrit sert à décrire l'élément patrimonial et à faire connaître le contexte historique ; la photo permet d'évoquer le patrimoine matériel (sites naturels, bâtiments, artéfacts) ; l'enregistrement sonore capte la mémoire des lieux et les archives orales ; la vidéo évoque efficacement le caractère vivant du patrimoine culturel immatériel (fêtes, festivals, pratiques artisanales et artistiques) ; et les animations 3D représentent de manière ludique l'évolution physique des sites.

Les éléments textuels sont divisés en deux parties : d'abord, une courte description générale d'environ 100 mots dévoile l'élément patrimonial et, ensuite, un texte plus élaboré d'environ 400 à 800 mots traite de l'élément patrimonial, soit sa nature, son histoire, sa patrimonialisation. De plus, on peut lire une introduction générale sur le patrimoine des francophones de l'ensemble du Canada de même qu'un texte d'introduction pour chacune des dix provinces et chacun des trois territoires. Plus longues, généralement de 1500 à 2000 mots, ces diverses introductions des provinces et des territoires sont axées sur l'histoire et la situation actuelle de la francophonie dans le territoire en question.

Ces écrits, qui évoquent la grande diversité des francophonies et de leurs patrimoines, sont accompagnés de photos qui illustrent des aspects actuels du site et des éléments de son histoire. La vidéo est exploitée pour transmettre la vitalité des pratiques traditionnelles des francophones, notamment leur patrimoine immatériel (festivals, chanson, musique, danse). Des capsules vidéo d'une à deux minutes présentent, sans narration, chacune des provinces à l'aide d'images marquantes et de musiques attrayantes. Environ la moitié des sites, soit 75 sur un total de 150, com- 
porte une vidéo plus longue, de 4 à 5 minutes, afin de faire découvrir la mémoire du lieu et les éléments forts de son patrimoine culturel immatériel. Ces vidéos, basées sur une ou deux entrevues de type ethnologique, sont rehaussées par l'insertion de photos ou de courts extraits vidéo visant à éclairer les propos des informateurs et à dynamiser l'entrevue.

Environ quarante sites sont accompagnés d'archives orales. Plutôt que de présenter des documents d'archives sous forme écrite, nous avons sollicité des étudiants de théâtre pour lire à haute voix ces textes marquants de l'histoire des francophones - extraits de discours d'hommes politiques, de textes de lois, de journaux privés, de chansons, de poèmes, etc. - afin de donner au programme interprétatif un caractère plus vivant et dynamique. Toujours avec le souci d'animer l'histoire, nous avons reconstitué, par un procédé numérique 3D, l'évolution historique depuis la fondation de cinq sites patrimoniaux jugés significatifs : Louisbourg (Nouvelle-Écosse), Pointeà-Callière (Québec), Sainte-Marie-au-pays-des-Hurons (Ontario), la Cathédrale de Saint-Boniface (Manitoba) et la Mission du Lac La Biche (Alberta) ${ }^{1}$. Très convivial, le site web permet une navigation facile entre les informations complémentaires des différents médias et la constitution de circuits de visite, soit par thème, sous l'onglet « Découvrir » (sites patrimoniaux, gastronomie, festivals et événements culturels, etc.), ou par zone géographique, sous l'onglet « Où aller» (province ou territoires). Le site est disponible en français et en anglais, et il se trouve à l'adresse suivante : corridorcanada.ca.

Il s'agit d'un outil d'interprétation novateur qui interprète pour la première fois en ligne les sites patrimoniaux de l'ensemble de la Francophonie canadienne. Lors du lancement officiel à Ottawa, le 20 février 2018, Mélanie Joly, ministre du Patrimoine canadien a souligné que « Le Corridor est l'un des projets phares que nous avons mis de l'avant pour promouvoir la francophonie canadienne dans le cadre des célébrations du $150^{\mathrm{e}}$ anniversaire de la fondation du Canada 150. Il constitue un legs majeur pour les francophones et francophiles du Canada. Ce projet est une reconnaissance sans équivoque du patrimoine francophone de notre pays. Il fait connaître des produits locaux et propose des expériences uniques. C'est aussi une façon novatrice de présenter la francophonie canadienne au reste du monde. »

La CRCP et le LEEM ont eu la chance et l'honneur d'être sélectionnés pour réaliser le contenu textuel et multimédia du projet. La coordination, la mise en œuvre et la pérennité du Corridor ont été confiées au RDÉE Canada. Le Corridor a permis à une quinzaine de nos professionnels et de nos étudiants de travailler à ce projet pendant plus de dix-huit mois. Il va sans dire que nous avons bénéficié d'une forte et précieuse collaboration des communautés francophones d'un bout à l'autre du pays.

\section{Médiations numériques du patrimoine}

L'obtention d'une importante subvention de 200000 \$ de la Fondation canadienne pour l'innovation en 2016 pour le projet « Explorer et vivre l'esprit du lieu par la réalité virtuelle et la réalité augmentée : médiations numériques du patrimoine » nous a permis l'embauche d'un professionnel à temps partiel et l'achat d'équipement destiné

1. À titre d'exemple de ces animations 3D, voir celle de la Mission du Lac La Biche : corridorcanada.ca/resource/lac-la-biche-un-site-historique-du-moyen-nord-qui-vaut-le-detour/\#group-2. 
à faire de la réalité virtuelle et de la réalité augmentée (caméras et microphones pour la captation en 3D, logiciels d'assemblage d'images et de son, casques, scanneurs et imprimantes 3D, par exemple). Cet équipement de pointe permettra au LEEM de passer des modalités d'interprétation du patrimoine en $2 \mathrm{D}$ à l'interprétation en $3 \mathrm{D}$, une tendance qui deviendra la norme d'ici quelques années. Le projet est guidé par quatre objectifs : répondre à une demande croissante de recherche et de création dans les médiations numériques en 3D des patrimoines à l'international ; mettre en place des infrastructures de pointe destinées à créer des contenus sur les patrimoines en réalité virtuelle et en réalité augmentée de manière expérimentale et à étudier les usages que l'on en fait par des méthodes de participation et d'observation variées ; offrir aux postdoctorants, aux étudiants-chercheurs et aux stagiaires une formation de pointe ; faire du Québec et du Canada un leader dans la médiation en réalité virtuelle des patrimoines, un secteur qui connaîtra sous peu un développement phénoménal. Sur le plan théorique, le laboratoire vise à éclairer et à mieux comprendre le rapport entre le patrimoine matériel et le patrimoine immatériel, entre les objets et espaces matériels et le sens que les humains leur donnent. Nous partons de l'hypothèse que cette relation entre le matériel et l'immatériel construit " l'esprit du lieu », concept recteur de notre projet. De plus, la réalité virtuelle permettra au LEEM de tester concrètement différentes hypothèses issues de sa réflexion sur le transmédia et la narrativité appliquée à la médiation des patrimoines. Il est également possible d'intégrer des contenus multimédias à même l'environnement virtuel immersif. Le laboratoire pourra alors observer les rapports entre immatériel et matériel dans un même espace qui reproduit fidèlement l'esprit du lieu. Le récit créé par la mise en commun de ces éléments du patrimoine favorisera la réception des contenus et le processus d'appropriation chez l'utilisateur.

\section{Diffusion en réalité virtuelle du patrimoine historique de Sillery}

Ce projet de diffusion numérique par la réalité virtuelle vise à mettre en valeur de manière innovatrice et vivante le patrimoine historique de Sillery dans le cadre du projet d'interprétation en ligne de la Ville de Québec intitulé « Découvrir Québec ». Le patrimoine peu et mal connu de l'arrondissement historique de Sillery a vu pourtant se succéder plusieurs étapes cruciales de la construction du Canada: des occupations amérindiennes préhistoriques, l'édification des premières missions jésuites et des seigneuries de la Nouvelle-France, des grands domaines des barons du bois britanniques, les résidences des gouverneurs du Canada-Uni et du premier gouverneur du Canada (Lord Monk), des quartiers ouvriers irlandais, des résidences et institutions d'enseignement catholiques canadiennes-françaises et québécoises du $\mathrm{Xx}^{\mathrm{e}}$ siècle et des cimetières multiethniques actuels. En s'appuyant sur les résultats des recherches menées précédemment par la Société historique de Sillery (SHS) et par l'Université Laval, le LEEM projette d'élaborer un outil d'interprétation virtuelle afin d'offrir au plus large public une expérience inédite de proximité et d'interactivité avec ce chapitre important de l'histoire canadienne. Les contenus détenus par la SHs (textes de présentation des biens patrimoniaux, archives orales, photos contemporaines et d'archives, entrevues vidéo, etc.) et la Ville de Québec seront déployés dans le site web adaptatif, « Découvrir Québec ». Le programme interprétatif comprendra une 
reconstitution virtuelle 3D du site de la Maison des Jésuites. Le projet est financé en partie par la Ville de Québec et par une subvention du Conseil de recherches en sciences humaines du Canada (CRSH), et sera lancé en septembre 2018.

\section{L'inventaire multimédia du patrimoine religieux de l'île d'Orléans}

Mettant à profit son expertise en inventorisation du patrimoine et ses équipements technologiques de pointe, le LEEM participe depuis 2017 à un projet qui vise à mieux connaître et à sauvegarder le patrimoine mobilier et immatériel religieux de l'île d'Orléans. Dans le cadre d'un partenariat entre le LEEM et le Comité du patrimoine religieux de l'île d'Orléans, un inventaire multimédia en ligne est en cours de réalisation dans les églises de ce site patrimonial unique. Le projet permettra de documenter une collection aujourd'hui méconnue et menacée malgré son ancienneté et sa richesse, puis de mettre en valeur les œuvres d'art, pièces d'orfèvrerie, meubles, documents et vêtements liturgiques présentant un intérêt historique, artistique ou ethnologique. Grâce à des enquêtes orales, il permettra également de recueillir la mémoire vivante des Orléanais et de recenser des éléments du patrimoine immatériel, tels des savoirfaire liés aux usages des objets ou des pratiques culturelles et rituelles liées au culte. Afin de diffuser les connaissances et de sensibiliser le grand public au patrimoine religieux, les résultats de l'inventaire seront accessibles en ligne, notamment sur le Répertoire du patrimoine culturel du Québec. En plus de textes informatifs, les fiches d'inventaires présenteront des contenus multimédias (photos 2D et 3D, vidéos) produits sur le terrain par les recherchistes associés au LEEM (Benoît Vaillancourt et Florence Gagnon-Brouillet). Réalisé sous les auspices du Comité du patrimoine religieux de l'île d'Orléans (COPRIO), ce projet bénéficie du soutien financier du ministère de la Culture et des communications du Québec, du Diocèse de Québec, des fabriques de Sainte-Trinité-d'Orléans et de la Sainte-Famille-d'Orléans.

\section{Université d'été internationale}

La Chaire et le LEEM ont organisé une université d'été internationale consacrée à la sauvegarde et à la mise en valeur du patrimoine religieux de l'île d'Orléans du 5 au 12 mai 2018, avec la collaboration du CopRIO, et en partenariat avec le programme de master en patrimoine de l'Université Jean-Monnet de Saint-Étienne (France). Cette formation créditée a regroupé environ 25 étudiants des $1^{\mathrm{er}}, 2^{\mathrm{e}}$ et $3^{\mathrm{e}}$ cycles ainsi qu'une dizaine de professionnels et de professeurs, dont des spécialistes du patrimoine religieux : John Porter, Jean Simard, $M^{\text {gr }}$ Marc Pelchat, Louis Painchaud, Madeleine Landry, France Lachance, ainsi que le professeur Robert Belot de l'Université JeanMonnet de Saint-Étienne, sa collègue Aurélie Brayet et cinq de leurs étudiants, Les professeurs et les étudiants français ont pu y participer grâce à un projet de collaboration financé dans le cadre de la Coopération France-Québec. L'Université d'été est une formule pédagogique et méthodologique innovante d'enseignement et de recherche universitaire qui vise à rapprocher du milieu les étudiants, les professeurs et les professionnels de recherche par l'organisation de formations in situ en collaboration, voire en partenariat, avec des intervenants locaux. Il s'agit d'amener les étudiants et les professeurs sur le terrain pour travailler sur des enjeux de société (ici le patrimoine religieux) avec les organisations concernées (ici les églises, les institutions culturelles 
et les municipalités) afin de stimuler les échanges, interactions et les transferts de connaissances, puis d'apporter des solutions aux problèmes identifiés. La formation est intensive (sur une semaine) et elle est à la fois théorique et pratique. Des conférences en début de formation apportent des éléments théoriques et comparatifs à la problématique, et les travaux de terrain visent à réaliser des actions concrètes pour la sauvegarde et la mise en valeur du patrimoine religieux. Les étudiants, travaillant en équipe de deux ou de trois et encadrés par les professeurs et des professionnels, ont réalisé neuf projets : un inventaire de biens religieux à l'église de Sainte-Famille ; un inventaire de biens religieux à l'église de Sainte-Pétronille; la conception d'un site web pour diffuser les résultats des travaux d'inventaire du patrimoine religieux de l'île d'Orléans; une vidéo sur la nature et les usages des vêtements liturgiques de l'église de Saint-Laurent; une vidéo sur la restauration des objets religieux (Centre de conservation du Québec) ; une vidéo sur la nature et les usages de l'orfèvrerie (église de Saint-Laurent) ; un programme d'interprétation en 3D de la Chapelle anglicane à l'aide de la panophotographie ; un programme d'interprétation du patrimoine religieux de l'église de Sainte-Famille; et un guide des bonnes pratiques de mise en valeur des patrimoines pour les municipalités. L'expérience a été très concluante pour tous les groupes concernés.

\section{Pour une nouvelle gouvernance des musées}

Laurier Turgeon lance cette année un nouveau projet triennal sur la gouvernance des musées, avec Yves Bergeron de l'UQÀM et Habib Saïdi de l’Université Laval, financé par le CRSH. Ce sujet est devenu aujourd'hui un enjeu majeur aussi bien dans les recherches scientifiques que pour les politiques culturelles et patrimoniales. Les musées vivent des transformations structurales importantes caractérisées par la présence de plus en plus marquée d'institutions ou de collections muséales parapubliques ou privées, la diversification des modalités d'accès au musée, l'approche touristique de la visite, la prédominance du numérique dans la mise en exposition des objets et dans la communication avec les visiteurs. À cette liste déjà longue, s'ajoute la tendance forte des chercheurs et des visiteurs à remettre en cause le pouvoir de l'État et des organisations internationales dans la gestion du patrimoine afin de favoriser plutôt des modes de gestion communautaires et participatifs où le musée sert de « zone de contact» entre les différents acteurs, porteurs, transmetteurs et récepteurs de ce patrimoine.

Ces importantes mutations que connaissent les musées entraînent les administrateurs et les directeurs à revoir entièrement les modes de prises de décisions, les structures de gestion, les politiques d'acquisition et d'exposition, et le fonctionnement même de l'institution. En effet, ces changements forcent à repenser la gouvernance du musée à travers le triple prisme de la démocratie, de la participation citoyenne et de l'ouverture au monde qui sont à la base de sa création. Ce constat est d'autant plus significatif que la problématique de la diversité culturelle, soutenue par la Convention sur la protection et la promotion de la diversité des expressions culturelles adoptée par l'UNESCO en 2005 et ratifiée par le Canada en 2006, est devenue un enjeu mondial pour les musées et plus particulièrement pour le Canada et le Québec qui comptent de nombreuses communautés culturelles, mais qui sont peu représentées dans les 
collections muséales. L'avenir des institutions muséales repose de plus en plus sur les règles de gouvernance et les rapports que les musées entretiennent avec leurs communautés (citoyens, artistes, représentants politiques, mécènes, bénévoles). La gouvernance des musées représente également un domaine de recherche intersectoriel, au carrefour des préoccupations de la gestion et du patrimoine, de la démocratie participative et de la culture publique, croisant souvent plusieurs champs d'intérêts d'institutions culturelles internationales, nationales, territoriales et associatives.

Ce domaine de recherche neuf exige une méthodologie propre, adaptée à la spécificité de l'objet d'étude. La méthodologie privilégiée par ce projet repose sur une démarche interdisciplinaire et intersectorielle qui vise à mettre à contribution et à croiser les travaux des spécialistes de la muséologie et du patrimoine et les intervenants du milieu. Il s'agit de mobiliser et de croiser quatre approches et quatre catégories de sources : l'approche historique en menant une étude critique à la fois à partir de travaux déjà réalisés sur l'administration des musées et des corpus documentaires des musées (procès-verbaux de conseils d'administration, rapports, mémoires, notes de service, etc.) ; l'approche d'analyse de contenu des textes de nature plus politique et juridique (conventions internationales, chartes, déclarations, textes de loi, politiques, codes d'éthique) ; l'approche ethnologique en menant des enquêtes de terrain dans les musées auprès d'administrateurs, de professionnels et de visiteurs (entrevues semi-dirigées, observation directe, groupes de discussion); et l'approche proprement muséologique par l'étude des pratiques muséales (les modalités de prises de décision, la participation des publics, l'observation de l'interactivité entre les œuvres exposées et les visiteurs, l'évaluation des expositions). Nous souhaitons aboutir à l'élaboration d'un modèle théorique qui permettra de prendre en charge ces quatre perspectives.

\section{Prix d'excellence 2017 pour l'encadrement des étudiants}

Laurier Turgeon a obtenu le Prix d'excellence pour l'encadrement aux cycles supérieurs 2017 de l'Université Laval pour son dossier exceptionnel en matière d'encadrement aux cycles supérieurs. Pour plus de détails au sujet de ce prix, on se reportera plus haut à la rubrique « Prix, honneurs et distinctions ».

LAURIER TURGEON

\section{Maison des arts de la parole}

138 , rue Wellington Nord, $2^{\mathrm{e}}$ étage

Sherbrooke (Québec)

J1H 5C5

Téléphone : (819) 566-6996

Télécopieur : (819) 780-8972

Courriel : info@maisondesartsdelaparole.com

Toile : www.maisondesartsdelaparole.com

\section{Une année chrysalide}

L'année 2017-2018 en fut une de changements, de défis et de réalisation pour la Maison des arts de la parole. Avec, à la fin de l'été, le départ en congé de maternité de la directrice générale, et donc la passation à une remplaçante, l'embauche d'une 